\title{
Sexual and gender-based violence and HIV in South Africa: An HIV facility-based study
}

\author{
L Artz, ${ }^{1}$ PhD; L Klazinga; ${ }^{2}$ A Müller, ${ }^{1}$ Dr med \\ ${ }^{1}$ Gender Health and Justice Research Unit, Division of Forensic Pathology, Department of Pathology, Faculty of Health Sciences, University of \\ Cape Town, South Africa \\ ${ }^{2}$ AIDS Healthcare Foundation, Durban, South Africa
}

Corresponding author: L Artz (Lillian.artz@uct.ac.za)

\begin{abstract}
Background. South Africa (SA) is known to have high levels of sexual and gender-based violence (SGBV) and HIV. Studies that explore the intersections of the two phenomena tend to be done at sites that provide services for survivors of SGBV, but few have explored experiences of SGBV of individuals attending HIV testing and treatment health facilities. Although HIV voluntary counselling and testing (VCT) services are generally well practised at ensuring pre- and-post-test counselling, there is little evidence that SGBV is included in routine screening. There is therefore a gap in knowledge of the prevalence of SGBV among patient populations in HIV testing and treatment settings. Objectives. To assess levels of SGBV in a patient population of an HIV facility in SA.

Methods. This cross-sectional study used a screening tool and a longer in-depth questionnaire on the prevalence of SGBV. A random sample of VCT clients were screened. Prevalence questionnaires were administered to a convenience sample of patients attending treatment literacy classes.

Results. A total of 1936 VCT clients completed the screening tool, of whom $2 \%$ reported forced sex without a condom or rape. Of the 436 patients who completed the in-depth prevalence questionnaire, $71 \%$ were women; $12 \%$ of women had been forced to have sex the last time that they had sexual intercourse. The lifetime prevalence of sexual violence was $14 \%$ and the lifetime prevalence of physical violence was $16 \%$. Conclusions. Levels of SGBV are high among populations attending HIV testing and treatment facilities. Healthcare providers need to be able to identify SGBV and provide appropriate services to survivors of SGBV who are seen at HIV testing and treatment facilities.
\end{abstract}

S Afr Med J 2020;110(5):377-381. https://doi.org/10.7196/SAMJ.2020.v110i5.13942

Among the 7.9 million people living with HIV in South Africa (SA) in $2017, \sim 60 \%$ are women, with young women aged $15-24$ years having an HIV incidence rate four times higher than males of the same age, highlighting the increasing 'feminisation' of the HIV epidemic. ${ }^{[1]}$ Young women are particularly vulnerable to HIV infection and sexual and gender-based violence (SGBV), as many of them experience poverty and economic insecurity, and have limited sexual agency. ${ }^{[2]}$ The degenerative impact of HIV/AIDS is profound, with multiple sexual partnerships, age-disparate relationships, inconsistent contraceptive use and stigma cited as the main drivers of the epidemic. ${ }^{[3]}$

SGBV itself is a risk factor for HIV. As a result of SGBV, women especially are vulnerable to HIV infection in complex and intertwined ways, ranging from coercive intimate-partner sexual intercourse without the use of a condom to rape. The World Health Organization (WHO) identified several direct and indirect SGBV-linked sources of HIV transmission: (i) direct transmission through sexual violence, including forced or coercive sexual intercourse with an HIV-infected partner; (ii) indirect transmission through sexual risk-taking, with studies supporting the theory that women's experience of violence is linked to increased risk-taking, including having multiple partners or non-primary partners (or partnerships outside marriage), or engaging in transactional sex; (iii) indirect transmission through inability to negotiate condom use; (iv) indirect transmission by partnering with riskier/older men, sometimes leading to the use of violence in the process of negotiating condom use; and ( $v$ SGBV as a consequence of being HIV-positive or fear of violence as a barrier to disclosure of HIV status among those women who do seek testing. ${ }^{[4]}$ SGBV is therefore a risk factor for HIV infection, and HIV infection is a risk factor for
SGBV. Although HIV infection as a consequence of violence against women is sufficiently covered by current research, serious gaps exist with respect to prevalence rates of SGBV among individuals seeking voluntary counselling and testing (VCT) services. ${ }^{[5-7]}$

Despite the clear mutually compounding link between HIV and SGBV, services for, and research on, SGBV have suffered from: (i) fractured and delayed health responses to women who have acquired HIV through sexual violence; (ii) lack of any prevalence rates of SGBV among individuals seeking HIV VCT services in SA; (iii) lack of availability of information and data to effectively inform programming, including poorly defined health 'indicators' for SGBV; and (iv) lack of coherent, systematic and reliable surveillance systems for SGBV data collection at VCT centres and HIV clinics. Currently, there is no comprehensive screening tool that screens women and men at VCT facilities for both intimate-partner violence and nonintimate-partner sexual violence. One notable exception is the work of Hassen and Deyassa, ${ }^{[8]}$ who examined both forms of violence among women using VCT services in South Wollo Zone, Ethiopia, and found a relationship between sexual violence and HIV infection. Using a modified WHO questionnaire, they studied the prevalence of lifetime sexual violence, lifetime intimate-partner violence and past 12 months intimate-partner violence, finding prevalence rates of $34.6 \%, 32.3 \%$ and $10.5 \%$, respectively. Both intimate-partner violence and lifetime sexual violence by another perpetrator were associated with HIV infection.

Other research investigating the link between sexual violence and HIV has generally been conducted on populations of sexual assault survivors who present at medicolegal facilities. ${ }^{[9]}$ While this research explores sexual violence by both intimate partners and non-intimate partners, the populations under study are known sexual assault 
survivors seeking medicolegal services - in other words, survivors who have officially reported a sexual assault. It is estimated, however, that only one in nine survivors of sexual violence reports the assault. Research done with survivors at medicolegal facilities may therefore underestimate the prevalence of SGBV. The WHO has identified that further research should assess whether and how asking women about intimate-partner violence in the context of HIV VCT is feasible. ${ }^{[10]}$

The development of a rapid and reliable violence screening instrument at VCT facilities could play a significant role in identifying survivors of SGBV. Identifying survivors would allow HIV providers to recognise specific counselling or health needs of survivors, and thus to tailor their comprehensive HIV and general healthcare response, particularly to women.

\section{Objectives}

To assess levels of SGBV in a patient population of an HIV facility in SA.

\section{Methods}

Drawing on the methodology of Hassen and Deyassa, ${ }^{[8]}$ we conducted a facility-based cross-sectional study on SGBV and HIV, which examined the incidence, prevalence and nature of intimatepartner violence and non-intimate-partner sexual violence in the clinic population at the AIDS Healthcare Foundation (AHF) clinic in Umlazi, KwaZulu-Natal (KZN) Province. The clinic provides comprehensive HIV services to the general population, including VCT, HIV treatment, treatment literacy classes and adherence counselling. We screened all patients coming for VCT for SGBV. Additionally, we measured the prevalence of sexual violence among a group of patients participating in treatment literacy classes. We included women and men in the study in order to gain a more comprehensive picture of the extent and nature of SGBV among HIV testing and treatment patients more generally.

\section{Instruments and sampling}

The recruitment process involved a rigorous informed and voluntary consent process. We designed two instruments to complement the existing screening and assessment forms used by HIV counsellors and clinicians as part of current screening protocols. The survey consisted of two sections: (i) a brief screening questionnaire pertaining to the reasons for being tested, specifically asking about SGBV; and (ii) an anonymous SGBV prevalence questionnaire assessing current and past experiences of sexual and other forms of violence as survivor or perpetrator.

The brief screening questionnaire was incorporated into the facility's existing consent, information, pre-test counselling and screening protocol for all VCT clients, adding the following questions: (i) whether the patient was seeking testing because they were forced or coerced to have sexual intercourse without a condom; and (ii) whether they were sexually assaulted/raped. If either (i) or (ii) had occurred, the patient was asked when it occurred, whether they had undergone a medicolegal examination, whether it had been reported to the police, whether the patient received counselling for the incident, and if not, whether they would like to receive any of the services inquired about.

The prevalence questionnaire asked patients who had tested HIV-positive and were attending adherence training or treatment literacy sessions about the prevalence of sexual violence at last sexual intercourse and over the respondent's lifetime, including asking for sociodemographic information and about perpetrators of violence. The questions relating to experiences of SGBV were guided by the WHO's guided questions on Intersections of Intimate Partner Violence. ${ }^{[4]}$ The questions covered the following HIV transmission risks: (i) direct transmission through sexual violence; (ii) indirect transmission through sexual risk-taking; (iii) indirect transmission through inability to negotiate condom use; and (iv) violence as a consequence of living with HIV.

Both questionnaires were reviewed and revised by clinic staff and piloted. Clinic staff were trained on using the screening instrument and the prevalence questionnaire, as well as on eligibility criteria, confidentiality and research ethics. Additionally, staff were trained on the legal framework for sexual offences and domestic violence (the Criminal Law (Sexual Offences and Related Matters) Amendment Act 32 of 2007, hereinafter referred to as the Sexual Offences Act, as well as the Domestic Violence Act 116 of 1998), including existing protocols; the public health, social and structural contexts of sexual offences and other forms of gender-based violence; and the referral protocol for patients seeking further care or support related to current or previous violence. Data collection took place between November 2016 and April 2017.

A total of 1936 clients completed the brief screening questionnaire, and 436 completed the in-depth prevalence questionnaire.

\section{Inclusion and exclusion criteria}

Inclusion criteria for participation in the screening phase included all individuals aged $>18$ years who attended the clinic for testing or counselling services as part of the AHF's standard protocol, after providing informed consent to VCT services. The second phase (prevalence questionnaire distributed during treatment literacy training) included consenting participants aged $>18$ years who attended the treatment literacy training and took part in psychosocial services programmes at the facility.

\section{Data analysis}

Descriptive data analysis was done using Stata 13 (StataCorp, USA).

\section{Process of obtaining informed consent}

The screening tool was added to the existing AHF pre-testing screening protocol. Consent is obtained as an integral part of the clinic's standard of care, in the course of which patients are given information on the privacy and confidentiality processes effective at the clinic. A separate research consent form was used for the prevalence phase of the study. The study was approved by the University of Cape Town's Faculty of Health Sciences Human Research Ethics Committee (ref. no. 064/2016). The research protocol complies with the South African National Health Act 61 of 2003 and with the latest version of the Declaration of Helsinki. ${ }^{[11]}$

\section{Results \\ VCT population: Coercion and sexual violence as reason for HIV testing}

A total of 1936 people who came to the health facility for HIV VCT were screened. Of these, $1 \%(n=20)$ said that they had specifically come for VCT because they had been coerced into having sex without a condom. Another $1 \%(n=14)$ said that they had come for VCT because they had experienced sexual violence or been raped.

\section{Treatment literacy population: Sexual behaviour and prevalence of forced sex}

The 436 participants who had tested HIV-positive and were attending treatment literacy classes were interviewed by the clinic psychosocial counsellors. The participants' ages ranged from 18 to 70 years (mean 32 years), $71 \%$ were women, and the majority were single ( $89 \%$ ). Although $84 \%$ had matriculated from high school, only $38 \%$ were employed (Table 1). 
Table 2 shows the characteristics of participants' last sexual intercourse. Of note, $54 \%$ had not discussed condom use, and $62 \%$ had not used a condom. This did not differ between women and men. Sixteen percent of male participants had had sex with another man, and $2 \%$ of female participants had had sex with another woman.

Ten percent of all participants (44/428), and $12 \%$ of women (35/298), had been forced to have sex the last time that they had sexual intercourse, by either a partner or a non-partner. Of those, 7 had been forced to have sex by more than one person. Table 3 shows further details of forced sex at last intercourse.

\section{Treatment literacy population: Lifetime prevalence of sexual violence}

The lifetime prevalence of sexual violence was $14 \%$ among all participants who participated in the survey at the treatment literacy

\begin{tabular}{|c|c|}
\hline Age (years), mean (SD) & $32.4(8.9)$ \\
\hline \multicolumn{2}{|l|}{ Gender $(N=434), n(\%)$} \\
\hline Women & $306(70.5)$ \\
\hline Men & $128(29.5)$ \\
\hline \multicolumn{2}{|c|}{ Marital status $(N=419), n(\%)$} \\
\hline Single & $372(89.2)$ \\
\hline Married & $35(8.4)$ \\
\hline Divorced & $4(1.0)$ \\
\hline Widowed & $3(0.7)$ \\
\hline Other & $3(0.7)$ \\
\hline \multicolumn{2}{|c|}{ Level of education $(N=370), n(\%)$} \\
\hline Matric & $310(83.8)$ \\
\hline Beyond matric & $60(16.2)$ \\
\hline \multicolumn{2}{|c|}{ Employment $(N=410), n(\%)$} \\
\hline Not employed & $219(53.4)$ \\
\hline Employed & $154(37.6)$ \\
\hline Student & $37(9.0)$ \\
\hline
\end{tabular}

\section{Table 2. Characteristics of last sexual encounter}

\begin{tabular}{ll}
\hline & $\boldsymbol{n}(\%)$ \\
\hline Gender of partner, female participants $(N=306)$ & \\
Male partner & $299(97.7)$ \\
Female partner & $5(1.6)$ \\
Male and female partner & $2(0.7)$ \\
Gender of partner, male participants $(N=127)$ & \\
Male partner & $20(15.8)$ \\
Female partner & $107(84.3)$ \\
Two or more sexual partners & \\
All participants $(N=396)$ & $31(7.8)$ \\
Female participants $(N=276)$ & $12(4.4)$ \\
Male participants $(N=118)$ & $19(16.1)$ \\
Had discussed condom use & $196(45.8)$ \\
All participants $(N=428)$ & $142(47.3)$ \\
Female participants $(N=300)$ & $53(42.1)$ \\
Male participants $(N=126)$ & \\
Did use a condom & $165(38.3)$ \\
All participants $(N=431)$ & $116(38.5)$ \\
Female participants $(N=301)$ & $49(38.3)$ \\
Male participants $(N=128)$ &
\end{tabular}

group. Among female participants, it was 17\%: 1 in 6 women. Sexual violence perpetrated by intimate partners was the most common (lifetime prevalence 9\%), followed by sexual violence perpetrated by a person known to the participant (lifetime prevalence 7\%). Table 4 provides a detailed analysis of the perpetrators and time periods of sexual violence.

\section{Treatment literacy population: Lifetime prevalence of physical violence}

The lifetime prevalence of physical violence was $16 \%$ among all participants. Among female participants, it was 19\%: almost 1 in

\begin{tabular}{ll} 
Table 3. Forced sex at last sexual encounter & \\
\hline & $\boldsymbol{n}(\%)$ \\
\hline Forced to have sex by partner & \\
All participants $(N=425)$ & $23(7.1)$ \\
Female participants $(N=295)$ & $6(4.7)$ \\
Male participants $(N=128)$ & \\
Forced to have sex by non-partner & $21(5.0)$ \\
All participants $(N=420)$ & $16(5.5)$ \\
Female participants $(N=291)$ & $5(3.9)$ \\
Male participants $(N=127)$ & \\
Has forced partner to have sex & $8(1.9)$ \\
All participants $(N=415)$ & $5(1.7)$ \\
Female participants $(N=287)$ & $3(2.4)$ \\
Male participants $(N=126)$ & \\
Has forced non-partner to have sex & $7(1.7)$ \\
All participants $(N=406)$ & $5(1.8)$ \\
Female participants $(N=281)$ & $2(1.6)$ \\
Male participants $(N=123)$ &
\end{tabular}

\section{Table 4. Sexual violence: Lifetime prevalence and} perpetrators

\begin{tabular}{ll}
\hline & $\boldsymbol{n}(\%)$ \\
\hline Lifetime prevalence of sexual violence & \\
All participants $(N=410)$ & $58(14.2)$ \\
Female participants $(N=282)$ & $48(17.0)$
\end{tabular}

Perpetrators of sexual violence, all participants Intimate partner

Lifetime prevalence $(N=404)$

In childhood (age <16) $(N=395) \quad 11(2.8)$

Since age $16(N=369) \quad 13(3.5)$

In the past 12 months $(N=376)$

Ex-intimate partner

Lifetime prevalence

In childhood $($ age $<16)(N=388) \quad 1(0.3)$

Since age $16(N=373)$

In the past 12 months $(N=367)$

Someone known

Lifetime prevalence $27(6.8)$

In childhood (age <16) $(N=392) \quad 20(5.1)$

Since age $16(N=347)$

In the past 12 months $(N=347) \quad 8(2.3)$

A stranger

Lifetime prevalence $12(3.2)$

In childhood $($ age $<16)(N=379) \quad 8(2.1)$

Since age $16(N=345) \quad 8(2.3)$

In the past 12 months $(N=340)$ 
5 women. Like sexual violence, physical violence was most often perpetrated by an intimate partner (lifetime prevalence $12 \%$ ). Table 5 shows more detailed findings.

\section{Discussion}

Our cross-sectional study with two different populations attending an HIV health facility in KZN shows that the lifetime prevalence of sexual and physical violence experienced by the clinic population was high, although seemingly under-reported at the VCT stage. We compare our findings with the literature, and discuss the implications and recommendations for comprehensive HIV service provision.

Our screening tool showed that only $2 \%$ of clients coming for VCT said that they came to test because they had been coerced to have sex without a condom or had been raped. Both constitute an offence under the Sexual Offences Act. This number may seem small until it is seen in context: $2 \%$ equates to 34 clients over a period of 20 weeks, which means that the VCT staff sees more than one patient a week for VCT as a result of a sexual assault. This finding has important implications for additional SGBV-related support that these clients may need, which we address further on.

The prevalence questionnaire, on the other hand, found that 1 in 10 participants and 1 in 8 female participants had been forced to have sex the last time they had sexual intercourse. The lifetime prevalence of sexual violence of $14 \%$ among all participants and $17 \%$ among female participants - or 1 in 6 women - also indicates a concerningly high rate of SGBV, particularly with regard to levels of sexual violence perpetrated by intimate partners ( 1 in 10). The lifetime prevalence of sexual violence among women living with HIV in this study is higher than the estimated lifetime prevalence among women in the general population (16\% among women living with HIV in our study, compared with $5-12 \%$ of women in the general population interviewed by Jewkes and Abrahams ${ }^{[12]}$ ). Our findings confirm the link between SGBV and HIV, through which women are rendered more vulnerable to both SGBV and HIV. ${ }^{[4]}$

There are several potential explanations for the discrepancy in SGBV prevalence between the screening tool and the more in-depth prevalence questionnaire, some of which raise important considerations for identifying SGBV and providing services for SGBV survivors in HIV clinical settings. Firstly, the populations were different for the two instruments: the in-depth prevalence questionnaire was administered to people who were living with HIV, while the screening tool was used with all people coming for VCT, including those who tested negative. Given the known link between SGBV and HIV, ${ }^{[9]}$ we would expect a higher level of SGBV experiences in an exclusively HIV-positive population. Secondly, research shows that questionnaires that ask more detailed questions about violence tend to find higher SGBV prevalence levels. ${ }^{[13]}$ The prevalence questionnaire in our study asked much more detailed questions than the brief screening tool, which could further explain the discrepancy in SGBV prevalence. Lastly, and perhaps most importantly, it is very likely that participants' willingness to disclose SGBV experiences depends on their perception of and trust in the environment in which they disclose. Participants are more likely to disclose SGBV to a counsellor they know and trust (such as the counsellors allocated to lead and accompany the treatment literacy groups over several weeks), and they are more likely to disclose SGBV if they perceive that support and follow-up services are available. This again is more likely to be the case among participants who are already embedded in the clinic's psychosocial support services.

While it is well known that sexual offences are under-reported in the criminal justice sector, including in South African Police
Table 5. Physical violence: Lifetime prevalence and perpetrators

\begin{tabular}{lc}
\hline & $\boldsymbol{n}(\%)$ \\
\hline Lifetime prevalence of physical violence & \\
All participants $(N=361)$ & $59(16.3)$ \\
Female participants $(N=251)$ & $48(19.1)$ \\
Perpetrators of physical violence, all participants & \\
Intimate partner & $46(12.2)$ \\
Lifetime prevalence $(N=377)$ & $19(4.8)$ \\
In childhood (age $<16)(N=396)$ & $26(6.9)$ \\
Since age $16(N=375)$ & $24(6.4)$ \\
In the past 12 months $(\mathrm{n}=375)$ & \\
Ex-intimate partner & $16(4.4)$ \\
Lifetime prevalence $(N=362)$ & $9(2.4)$ \\
In childhood $($ age $<16)(N=379)$ & $7(1.9)$ \\
Since age $16(N=371)$ & $6(1.7)$ \\
In the past 12 months $(N=360)$ & \\
Someone known & $19(5.4)$ \\
Lifetime prevalence $(N=353)$ & $16(4.2)$ \\
In childhood $($ age $<16)(N=377)$ & $6(1.7)$ \\
Since age $16(N=350)$ & $6(1.7)$ \\
In the past 12 months $(N=353)$ & \\
A stranger & $8(2.3)$ \\
Lifetime prevalence $(N=346)$ & $4(1.1)$ \\
In childhood $($ age $<16)(N=369)$ & $3(0.9)$ \\
Since age $16(N=348)$ & $5(1.4)$ \\
In the past 12 months $(N=348)$ &
\end{tabular}

Service annual reports on crime statistics, there is a paucity of research analysing under-reporting of sexual offences in an HIV testing context. There are a number of possible reasons for such under-reporting, including: (i) lack of training or mechanisms for HIV counsellors to ask about (screen for) SGBV; (ii) the view that screening for SGBV is not part of, or important to, the VCT process, or beyond the purview of the role of the testing and treatment practitioner; (iii) a concern about the 'cultural appropriateness' of asking questions that are perceived to be 'a family matter', or too detailed about sexual activity or experiences; (iv) a narrow understanding of what constitutes 'coercive or forced sex' among research participants, or the view that there is no real benefit in disclosing SGBV in an HIV testing and treatment environment; and (v) after the trauma of a sexual assault, individuals attending HIV facilities may prefer to treat just the potential physical outcomes of coerced sex rather than the psychological impact of that experience.

Health services, particularly those focused on HIV, provide an important entry point for identifying and responding to women who have experienced sexual violence, where, for instance, healthcare providers can facilitate further services to women for care, treatment or support in relation to physical injuries, sexual and reproductive health problems associated with violence (e.g. sexually transmitted infections, pelvic pain, unwanted pregnancies), or referrals to legal, medicolegal or counselling services. The differences in the findings between the screening tool and the prevalence questionnaire in our study suggest that screening for SGBV may best be done among patients who are embedded in an ongoing clinic programme, such as treatment literacy training or adherence counselling. The longerterm linkage to the clinic provides an ideal opportunity for SGBVrelated services, including psychosocial support and specialised referrals for further support and treatment. Offering SGBV-related services necessitates that all healthcare staff who provide HIV-related 
care (from counsellors to nurses to medical staff) have appropriate training on how to identify SGBV, and how to provide support for SGBV survivors. In the words of Ellsberg and Betron, ${ }^{[14]}$ 'providers need to be trained to identify women living with violence, and to respond with appropriate care and referrals, making sure not to put women at increased risk for violence through their actions'.

\section{Conclusions}

We recommend that clinic staff follow the WHO clinical and policy guidelines for responding to sexual violence against women. ${ }^{[10]}$ Specific recommendations for healthcare providers in the SA context include:

- Become familiar with the legal framework, specifically the Sexual Offences Act of 2007 and the Children's Act 38 of 2005, which provide definitions of sexual violence and sexual assault and lay out legal obligations for healthcare providers.

- Actively seek out existing services for survivors of SGBV in the clinic catchment area, to which survivors of SGBV can be referred. This should be done together with a social worker, if there is one at the clinic. Services are likely to be provided by non-governmental organisations, and medicolegal services are available at Thuthuzela Care Centres. Building relationships with service providers at these centres makes it easier to refer patients for counselling and further support.

- Initiate regular and ongoing conversations about SGBV among clinic staff, recognising that some of them will be survivors of SGBV themselves.

- Challenge attitudes that trivialise or diminish SGBV among colleagues and patients, in order to build a respectful and encouraging environment for survivors to disclose their experiences of SGBV.

Finally, in addition to asking patients about experiences of SGBV, health practitioners should ensure that all clinic users know that postexposure prophylaxis is available at the facility, how it works and why they should access it, through both the consultation process and visible messaging within the facility.

\section{Declaration. None.}

Acknowledgements. The authors thank Elizabeth Lubanyana (social worker), Dr Bright Mhlongo, the AHF, the VCT and counselling staff at AHF clinic in Umlazi, and Terri Ford. Special thanks to Valerie Grand-Maison for her assistance in training AHF staff and general research support.

Author contributions. LA and AM conceptualised the study. LK oversaw data collection. AM analysed the data. LA drafted the manuscript. All authors revised and edited the manuscript.

Funding. The study was funded by the AHF. The funder had no influence on study design or data analysis.

\section{Conflicts of interest. None.}

1. Human Sciences Research Council and Department of Science and Technology. The Fifth South African National HIV Prevalence, Incidence, Behaviour and Communication Survey, 2017. Cape Town: HSRC Press, 2018. http://www.hsrc.ac.za/uploads/pageContent/9234/SABSSMV_Impact Assessment_Summary_ZA_ADS_cleared_PDFA4.pdf (accessed 24 March 2020).

2. Shisana O, Rehle T, Simbayi LC, et al. South African National HIV Prevalence, Incidence and Behaviour Survey, 2012. Cape Town: HSRC Press, 2014. http://www.hsrc.ac.za/en/researchBehaviour Survey, 2012. Cape Town: HST
outputs/view/6871 (accessed 24 March 2020)

outputs/view/6871 (accessed 24 March 2020).
3. Higgins JA, Hoffman S, Dworkin SL. Rethinking gender, heterosexual men, and women's Higgins JA, Hoffman S, Dworkin SL. Rethinking gender, heterosexual men, and women's
vulnerability to HIV/AIDS. Am J Public Health 2010;100(3):435-445. https://doi.org/10.2105/ AJPH.2009.159723

4. World Health Organization. Violence against Women and HIV/AIDS: Critical Intersections. Intimate partner violence and HIV/AIDS. Information Bulletin Series, No. 1. 2004. https://www. who.int/hac/techguidance/pht/InfoBulletinIntimatePartnerViolenceFinal.pdf (accessed 11 March 2020)

5. Outwater A, Abrahams N, Campbell JC. Women in South Africa: Intentional violence and HIV/AIDS: Intersections and prevention. J Black Studies 2005;35(4):135-154. https://doi. org/10.1177/0021934704265915

6. Leburu G, Phetlho-Thekisho N. Reviewing gender-based violence against women and HIV/AIDS as intersecting issues. Soc Work 2015;51(3):399-420. https://doi.org/10.15270/51-3-455

as intersecting issues. Soc Work 2015;51(3):399-420. https://doi.org/10.15270/51-3-455
7. Peltzer K, Pengpid S. Sexual abuse, violence and HIV risk among adolescents in South Africa. Gend Peltzer K, Pengpid S. Sexual abuse, violence and HIV risk among ado
Behav 2008;6(1):1462-1478. https://doi.org/10.4314/gab.v6i1.23360

8. Hassen F, Deyassa N. The relationship between sexual violence and human immunodeficiency virus (HIV) infection among women using voluntary counseling and testing services in South Wollo Zone, Ethiopia. BMC Res Notes 2013;6:271. https://doi.org/10.1186/1756-0500-6-271

9. Christofides N, Muirhead D, Jewkes R, Penn-Kekana L, Conco N. Including Post-Exposure Prophylaxis to Prevent HIV/AIDS into Post-Sexual Assault Health Services in South Africa: Cost and Cost Effectiveness of User Preferred Approaches to Provision. Pretoria: Medical Research Council, 2006. https://www.unicef.org/southafrica/SAF_resource_violencehivaids.pdf (accessed 11 March 2020).

10. World Health Organization. Responding to intimate partner violence and sexual violence against women: WHO clinical and policy guidelines. 2013. https://www.who.int/reproductivehealth/ women: WHO clinical and policy guidelines. 2013 . https://wWw.
publications/violence/9789241548595/en/ (accessed 11 March 2020).

publications/violence/9789241548595/en/ (accessed 11 March 2020).
11. World Medical Association. World Medical Association Declaration of Helsinki: Ethical Principles World Medical Association. World Medical Association Declaration of Helsinki: Ethical Principles
for Medical Research Involving Human Subjects. Bull World Health Organ 2001;79(4):373-374. https://www.who.int/bulletin/archives/79(4)373.pdf (accessed 11 March 2010).

12. Jewkes R, Abrahams N. The epidemiology of rape and sexual coercion in South Africa: An overview. Soc Sci Med 2002;55(7):1231-1244. https://doi.org/10.1016/S0277-9536(01)00242-8

13. Bachman R. Measuring Rape and Sexual Assault: Successive Approximations to Consensus. Washington, DC: National Academy of Sciences, 6 June 2012. https://www.semanticscholar.org/ paper/Measuring-Rape-and-Sexual-Assault\%3A-Successive-to-Bachman/5d779285d774590f885 03b6a2216a8ed2db9b2a (accessed 25 March 2020).

14. Ellsberg M, Betron M. Preventing gender-based violence and HIV: Lessons from the field. USAID, 2010:1-4. http://citeseerx.ist.psu.edu/viewdoc/download?doi=10.1.1.360.3964\&rep=rep 1 \&type=p df (accessed 11 March 2020).

Accepted 28 February 2020 